\title{
Nonlinear Wave Equation in Special Coordinates
}

\author{
Alexander SHERMENEV \\ Wave Research Center, Russian Academy of Sciences \\ 38, Vavilov Street, Moscow, 117942, Russia \\ E-mail: sher@orc.ru
}

This article is part of the Proceedings titled "Geometrical Mathods in Physics: Bialowieza XXI and XXII"

\begin{abstract}
Some classical types of nonlinear periodic wave motion are studied in special coordinates. In the case of cylinder coordinates, the usual perturbation techniques leads to the overdetermined systems of linear algebraic equations for unknown coefficients whose compatibility is key step of the investigation. Their solutions give solutions to the nonlinear wave equation which are periodic in time and found with the same accuracy as the nonlinear wave equation is derived. Expanding the potential for wave motion in Fourier series, we express explicitly the coefficients of the first two harmonics as quadratic polynomials of Bessel functions. One may speculate that the obtained expressions are only the first two terms of an exact solution to the nonlinear wave equations.
\end{abstract}

\section{Introduction}

Separation of variables is one of the most important and old methods for solving the wave equation

$$
-\varkappa^{2} \phi_{t t}+\triangle \phi=0
$$

for potential $\phi\left(x_{1}, x_{2}, x_{3}, t\right)$. The method was first used by L'Hospital in 1750. Because of boundary conditions, a success requires choice of an appropriate coordinate system. The wave equation can be solved by separation of variables in only 11 coordinate systems: Cartesian, confocal ellipsoidal, confocal paraboloidal, conical, cylindrical, elliptic cylindrical, oblate spheroidal, paraboloidal, parabolic cylindrical, prolate spheroidal, and spherical coordinates (Eisenhart 1934).

Our general aim is to generalize the method of separation of variables for nonlinear wave equation:

$$
-\varkappa^{2} \phi_{t t}+\triangle \phi+\frac{1}{2} \alpha(\nabla \phi \cdot \nabla \phi)_{t}+\frac{1}{2} \beta\left(\phi_{t}^{2}\right)_{t}=0
$$

considered in the 11 special coordinate systems. In this communication, we focus our attention on the case of cylindrical coordinates. The cases of elliptic cylindrical and parabolic 
cylindrical coordinates, where Mathieu functions and functions of parabolic cylinder are involved, are essentially more difficult.

In the case of Cartesian coordinates, we can calculate the quadratic nonlinear correction to any monochromatic solution of (1.1). However for cylinder coordinates, an attempt to find a quadratic correction in terms of quadratic expressions of Bessel functions inevitably leads to the overdetermined systems of linear algebraic equations for unknown coefficients. The main author's result is that these overdetermined systems are compatible in a series of important cases which allow to construct explicit solution of (1.2) with the same accuracy as the equation is derived.

In two-dimensional case, equation (1.2) is the shallow water equation (see [1]) describing the long surface water waves.

In three-dimensional case, this is the nonlinear acoustic wave equation describing the waves in an isentropic gas flow for the non-dissipative case (see [2], [3], and [4]).

\section{Shallow water equation}

Two classic types of nonlinear wave motion in shallow water are studied using the twodimensional version of (1.2).

The first type is the periodic nonlinear waves climbing a sloping beach. The center of vertical coordinate system is located in the intersection of line of still water and of sloping bottom.

The second type is the axisymmetric waves (caused by periodic source of energy) and the simplest unsymmetric waves propagating over a horizontal bottom and considered in the horizontal coordinate system.

\section{Waves on a sloping beach}

Computer algebra system is used for deriving and studying the high-order nonlinear surface wave equations for long periodic nonlinear waves climbing on a beach. Potential is expanded in Fourier series up to the fourth harmonic inclusively. Coefficients of these series are explicitly presented as polynomials of Bessel functions. One may speculate that the obtained expressions are the first terms of the expanded exact solution to the surface wave equation over a sloping bottom. The first fourth harmonics for waves over a sloping beach are given in [5], [6] and [7].

\section{Waves in horizontal polar coordinates}

In the classic book "Hydrodynamics", $\S \S 191-195$, [8] at least two special cases of long linear waves over a horizontal bottom in polar coordinates $(\theta, r)$ are considered. The first one is axisymmetric waves caused by periodic source of energy. The second one is the simplest unsymmetrical wave motion used there for describing the unsymmetrical waves in a circular basin. In this note we present two solutions of the nonlinear shallow water equations in polar coordinates which are next order corrections to these well known linear solutions. (Axisymmetric nonlinear waves were subject of numerical investigations in series of papers. See bibliography in [9])

The potential at the bottom is expanded in Fourier series in time: 


$$
\begin{aligned}
f(r, \theta, t)=\varepsilon^{2} U(r, \theta)+\varepsilon S^{1}(r, \theta) & \sin (\omega t)+\varepsilon C^{1}(r, \theta) \cos (\omega t) \\
& +\varepsilon^{2} S^{2}(r, \theta) \sin (2 \omega t)+\varepsilon^{2} C^{2}(r, \theta) \cos (2 \omega t)+\ldots
\end{aligned}
$$

The explicit expressions for functions $U, S^{1}, C^{1}, S^{2}$, and $C^{2}$ up to the order $\varepsilon^{2}$ are derived, which are the homogenous polynomials of Bessel functions and trigonometric functions of variable $\theta$. Their coefficients are polynomials in $r$. The two linear solutions used in the book of [8] are the following:

$$
\begin{aligned}
& f(r, \theta, t)=\varepsilon J_{0}(\omega r) \sin (\omega t)+\varepsilon Y_{0}(\omega r) \cos (\omega t), \\
& f(r, \theta, t)=\varepsilon J_{1}(\omega r) \cos \theta \sin (\omega t) .
\end{aligned}
$$

\section{Nonlinear axisymmetric waves with periodic source}

Below we denote by $S=S(r)$ and $C=C(r)$ two solutions of the equation $r Z_{r r}+Z_{r}+$ $\omega^{2} r Z=0$, and by $S^{\prime}$ and $C^{\prime}$ theirs derivatives. Functions $S$ and $C$ can be expressed as linear combination of Bessel functions.

$$
\begin{aligned}
f(r, t)=\varepsilon S \sin (\omega t)+\varepsilon C \cos (\omega t) & +\varepsilon^{2}\left(\frac{\omega}{2}\left(S^{2}-C^{2}\right)+\frac{3 \omega}{4} r\left(S S^{\prime}-C C^{\prime}\right)\right) \sin (2 \omega t) \\
+ & \varepsilon^{2}\left(\omega C S+\frac{3 \omega}{4} r\left(S C^{\prime}+S^{\prime} C\right)\right) \cos (2 \omega t)
\end{aligned}
$$

\section{Nonlinear unsymmetric case, standing waves}

$$
\begin{aligned}
& f(r, \theta, t)=\varepsilon C^{\prime} \cos \theta \cos (\omega t) \\
& \quad+\varepsilon^{2}\left(\frac{3 \omega^{3}}{8} C^{2}+\frac{3 \omega^{3}}{8} r C C^{\prime}-\frac{\omega}{4} C^{2}+\left(\frac{3 \omega^{3}}{8} r C C^{\prime}+\frac{\omega}{8} C^{2}\right) \cos 2 \theta\right) \sin (2 \omega t) .
\end{aligned}
$$

The presented nonlinear corrections alter dramatically the topology of contours of surface waves. In particular, the contours vary in time in contrast with Lamb solution and the surface is never flat.

\section{Nonlinear acoustic wave equation}

Three-dimensional nonlinear acoustic wave equation (1.2) for potential $\phi\left(x_{1}, x_{2}, x_{3}, t\right)$ describes the waves in an isentropic gas flow for the non-dissipative case (see , [2] [3] and $[4])$.

We present a series of its solutions of (1.2) in the cylinder coordinates $(\theta, r, z)$. These solutions describe periodic waves traveling in direction of axe $z$ and have the same accuracy as equation (1.2) is derived. Among other applications, they can be useful for describing the nonlinear wave motion in tubes, waveguides, and resonators or the nonlinear waves scattered by a cylinder or by a wedge. The linear versions of these solutions were studied and used in a series of classic books (see [8], [10], and [11]). The presented solutions give 
nonlinear corrections to the classical linear solutions describing the wave propagation in tubes and waveguides.

The potential $\varphi$ written in the cylinder coordinates is assumed to be expanded in Fourier series:

$$
\begin{aligned}
\varphi(r, \theta, z, t)=\varepsilon S_{1}(r, \theta) \sin (K z+\omega t) & +\varepsilon^{2} S_{2}(r, \theta) \sin (2 K z+2 \omega t) \\
& +\ldots+\varepsilon^{m} S_{m}(r, \theta) \sin (m K z+m \omega t)+\ldots
\end{aligned}
$$

The function $S_{1}(r, \theta)$ is periodic in $\theta$, and we assume that it can be presented as a Fourier series in respect to $\theta$ containing only four terms:

$$
S_{1}(r, \theta)=a_{0} J_{0}(k r)+a_{1} J_{1}(k r) \exp (i \theta)+a_{2} J_{2}(k r) \exp (2 i \theta)+a_{3} J_{3}(k r) \exp (3 i \theta)
$$

because they are considered to be the most important (also because we failed to find the general expression) and $k=\sqrt{\varkappa \omega^{2}-K^{2}}$ (see, for example [10]). We can assume that $k=1$ without a loss of generality.

The main result of this work consists in the explicit expressions (3.3) - (3.12) for the function $S_{2}$, which are homogenous polynomials of Bessel functions $J_{0}(k r)$ and $J_{1}(k r)$ and the trigonometric functions of angular variable $\theta$. Their coefficients are polynomials of $r^{-1}$ and $r$. These expressions give periodic solutions to equation (1.2) within the same accuracy as the equation is derived.

We seek a particular solution in the form

$$
\begin{aligned}
& S_{2}(r, \theta)=a_{0}^{2} M_{00}+a_{0} a_{1} M_{01} \exp (i \theta)+a_{0} a_{2} M_{02} \exp (2 i \theta)+a_{1}^{2} M_{11} \exp (2 i \theta) \\
& +a_{0} a_{3} M_{03} \exp (3 i \theta)+a_{1} a_{2} M_{12} \exp (3 i \theta)+a_{1} a_{3} M_{13} \exp (4 i \theta) \\
& +a_{2}^{2} M_{22} \exp (4 i \theta)+a_{2} a_{3} M_{23} \exp (5 i \theta)+a_{3}^{2} M_{33} \exp (6 i \theta)
\end{aligned}
$$

where $M_{i j}=Q_{i j 00} J_{0}^{2}+Q_{i j 01} J_{0} J_{1}+Q_{i j 11} J_{1}^{2}$ and $Q_{i j s l}$ are polynomials of $r^{-1}$ and $r$ with unknown coefficients.

The explicit expressions for $M_{i j}$ are following:

$$
\begin{aligned}
& M_{00}=-(\lambda-\mu) \frac{r}{2} J_{0} J_{1}+\mu J_{0}^{2} \\
& M_{01}=(\lambda-\mu)\left(\frac{r}{2} J_{0}^{2}-\frac{r}{2} J_{1}^{2}\right)+\mu J_{0} J_{1} \\
& M_{02}=(\lambda-\mu)\left(r J_{0} J_{1}\right)+\mu J_{0} J_{2} \\
& M_{11}=(\lambda-\mu)\left(\frac{r}{2} J_{0} J_{1}-\frac{1}{2} J_{1}^{2}\right)+\mu J_{1}^{2} \\
& M_{03}=(\lambda-\mu)\left(-\frac{r}{2} J_{0}^{2}+\left(\frac{2}{r}+\frac{r}{2}\right) J_{1}^{2}\right)+\mu J_{0} J_{3} \\
& M_{12}=(\lambda-\mu)\left(-\frac{r}{2} J_{0}^{2}+2 J_{0} J_{1}+\left(-\frac{2}{r}+\frac{r}{2}\right) J_{1}^{2}\right)+\mu J_{1} J_{2} \\
& M_{13}=(\lambda-\mu)\left(-J_{0}^{2}+\left(\frac{4}{r}-r\right) J_{0} J_{1}+\left(2-\frac{4}{r^{2}}\right) J_{1}^{2}\right)+\mu J_{1} J_{3}
\end{aligned}
$$




$$
\begin{aligned}
& M_{22}=(\lambda-\mu)\left(-J_{0}^{2}+\left(\frac{4}{r}-\frac{r}{2}\right) J_{0} J_{1}+\left(1-\frac{4}{r^{2}}\right) J_{1}^{2}\right)+\mu J_{2}^{2} \\
& M_{23}=(\lambda-\mu)\left(\left(-\frac{8}{r}+\frac{r}{2}\right) J_{0}^{2}+\left(-6+\frac{32}{r^{2}}\right) J_{0} J_{1}+\left(-\frac{32}{r^{3}}+\frac{10}{r}-\frac{r}{2}\right) J_{1}^{2}\right)+\mu J_{2} J_{3} \\
& M_{33}=(\lambda-\mu)\left(\left(2-\frac{24}{r^{2}}\right) J_{0}^{2}+\left(\frac{96}{r^{3}}-\frac{20}{r}+\frac{r}{2}\right) J_{0} J_{1}+\left(-\frac{5}{2}-\frac{96}{r^{4}}+\frac{32}{r^{2}}\right) J_{1}^{2}\right)+\mu J_{3}^{2}
\end{aligned}
$$

\section{Conclusions}

A series of nonlinear solutions (3.3) - (3.12) found with the accuracy of $\varepsilon^{2}$ to the nonlinear acoustic wave equation in the cylinder coordinates is presented. The potential $\varphi(r, \theta, z, t)$ is a bounded function. If $a_{1}=a_{2}=a_{3}=0$, it tends to zero when $r$ tends to infinity. The solution can be checked by substitution into equation (1.2).

The linear versions of these problems when the terms only of the first order in $\varepsilon$ are retained were studied in a number of classical books of (see [8], [10], and [11]).

The derived formulas are obtained by the method of unknown coefficients as solutions of some overdetermined systems of algebraic linear equations. The reason for their solvability remains obscure in the moment.

Nevertheless, I conjecture that more general solution $\varphi(r, \theta, z, t)$ can be found (with accuracy $\varepsilon^{2}$ ) in the following form:

$$
\begin{aligned}
(r, \theta, z, t) & =\varepsilon S_{1}(r, \theta) \sin (K z+\omega t)+\varepsilon^{2} S_{2}(r, \theta) \sin (2 K z+2 \omega t) \\
S_{1}(r, \theta) & =b_{0} J_{0}(r)+J_{1}(r)\left(b_{1} \cos \theta+c_{1} \sin \theta\right) \\
& +\ldots+J_{i}(r)\left(b_{i} \cos i \theta+c_{i} \sin i \theta\right)+\ldots \\
S_{2}(r, \theta) & =M_{0}+M_{1} \cos \theta+N_{1} \sin \theta+\ldots+M_{i} \cos i \theta+N_{i} \sin i \theta+\ldots
\end{aligned}
$$

where $M_{i}$ and $N_{i}$ are quadratic polynomials of Bessel functions: $M_{i}=Q_{i 00} J_{0}^{2}+Q_{i 01} J_{0} J_{1}+$ $Q_{i 11} J_{1}^{2}$ and $N_{i}=P_{i 00} J_{0}^{2}+P_{i 01} J_{0} J_{1}+P_{i 11} J_{1}^{2}$ whose coefficients $Q_{i 00}, Q_{i 01}, Q_{i 11}, P_{i 00}, P_{i 01}$, and $P_{i 11}$ are polynomials of $r$ and $r^{-1}$.

\section{References}

[1] Mei, C. C. The Applied Dynamics of Ocean Surface Waves. Wiley, 1983.

[2] Shermenev A. Nonlinear acoustical waves in tubes, Acta Acoustica (to appear).

[3] Soderholm, L. H. , On the Kuznetsov Equation and Higher Order Nonlinear Acoustic Equations, in Nonlinear Acoustics at the Turn of the Millennium, Proc. 15th International Symposium of Nonlinear Acoustics, Lauterborn, W., and Kurz T. (eds.), Melville, N.Y.: AIP (v. 524), pp. 141-144, 2000. 
[4] Wojcik, J. A new theoretical basis for numerical simulation of nonlinear acoustic fields, in Nonlinear Acoustics at the Turn of the Millennium, Proc. 15th International Symposium of Nonlinear Acoustics, Lauterborn, W., and Kurz T. (eds.), Melville, N.Y.: AIP (v. 524), pp. 133-136, 2000.

[5] Shermenev, A. \& Shermeneva, M. Long periodic waves on an even beach. Physical Review E, No. 5, 6000-6002, 2000.

[6] Shermenev, A. Nonlinear periodic waves on a beach. Geophysical and Astrophysical Fluid Dynamics, No. 1-2, 1-14, 2001.

[7] Shermenev A. Nonlinear periodic waves in shallow water, Proceedings of Conference for Symbolic and Numerical Scientific Computation, Lecture Notes in Computer Science, Springer (to appear).

[8] Lamb, H. Hydrodynamics. Sixth Ed., Cambridge Univ. Press, 1932.

[9] Vanden-Broeck, J.-M. Water waves and related free surface flows. In Free Surface Flows: IUTAM Symposium, Birmingham 2000, Kluwer, Dordrecht, 29-38, 2001.

[10] Rayleigh, W. Theory of Sound, McMillan, London, 1929.

[11] Skudrzyk, E. The Foundation of Acoustics, Springer - Verlag, Wien, New York,1971. 